\title{
Time-related morphometric studies on CD34 protein expression in the cerebral blood vessels after fatal brain contusions
}

\author{
Mariusz Kobek ${ }^{1}$, Zbigniew Jankowski ${ }^{2}$, Janusz Szala ${ }^{3}$, Zbigniew Gąszczyk-Ożarowski ${ }^{4}$, Artur Pałasz ${ }^{5}$, Rafał Skowronek ${ }^{1}$ \\ ${ }^{1}$ Department of Forensic Medicine and Toxicology, School of Medicine in Katowice, Medical University of Silesia in Katowice, \\ ${ }^{2}$ Department of Forensic Medicine, Medical University of Gdańsk, ${ }^{3}$ Institute of Materials Science, Silesian University of Technology, \\ ${ }^{4}$ Department of Forensic Medicine, Wrocław Medical University, ${ }^{5}$ Department of Histology, Chair of Histology and Embryology, \\ School of Medicine in Katowice, Medical University of Silesia in Katowice, Poland
}

\begin{abstract}
In our previous work, we have discussed the importance of neurofilaments in determination of the age of brain contusions. The purpose of this paper is to examine a possibility of angiogenesis-related assessment of the age of brain contusions by means of morphometric analysis of the CD34 expression in the contused sites. The researched material comprised 90 cases divided into nine groups according to the time of death: immediately on the spot, 12 hours, 24 hours, 2 days, 3 days, 4 days, 5 days, 6 days, and 7 days after the head trauma. Immunohistochemically stained CD34 antigen was evaluated quantitatively with the Met-llo application. The results were then tested statistically for any regularities. Generally, there was a tendency to increase both the number of blood vessels and blood vessels area fraction in time. The results of Kruskal-Wallis analysis showed that numbers of blood vessels were significantly different in the first seven days after the head trauma, but significance levels of specific sample pairs, as calculated by Dunn's test, were not statistically significant. Thus, the conclusion is that the proposed method is of no considerable value in determination of the age of brain contusions.
\end{abstract}

Key words: brain contusion, angiogenesis, morphometry, survival time.

\section{Introduction}

Public authorities enquiring any violent death need to verify the date of an alleged offence in the course of which fatal trauma was inflicted. Often medicolegal experts can lend them a helping hand and determine the age of an injury. This statement is true for intracranial injuries, including contusions of cerebral hemispheres.

High-energy blunt trauma to the head, irrespective of its circumstances, can cause focal damage to peripheral, cortical-subcortical regions of cerebral hemispheres, commonly known as brain contusions [19]. This kind of injury takes the form of 
hemorrhage and necrosis. As the time passes, these lesions undergo resorption and organization. Reparative processes that initiate these changes occur in healthy nerve tissue peripherally to a contusion [2]. Microscopically, they are manifested by proliferation of capillaries containing stimulated endothelial cells. This phenomenon is a morphological hallmark of angiogenesis.

Angiogenesis consists in formation of new capillaries in postnatal life. It develops in sites that have been damaged by a trauma or a disease. Judah Folkman discovered and described it in 1971 [4]. Angiogenesis is not to be confused with vasculogenesis. The latter occurs mainly in prenatal life though notable exceptions to this rule, e.g. postnatal vasculogenesis from progenitor cells, are known [3]. Angiogenesis is thought to be the key to formation of granulation tissue in the course of wound healing, ischemia, inflammation and neoplasia, especially of malignant tumors [3,27]. Therefore, links between angiogenesis and brain trauma, including those accompanied by ischemia, are worth exploring.

Mechanisms that regulate angiogenesis have been intensively researched in experiments with animals. Recently, it has been observed that increased levels of miR-126 (microRNA) both promote angiogenesis and inhibit leukocyte extravasation into the contused spinal cord. The process was accompanied by downregulation of mRNA and genes of SPRED1, PIK3R2 and VCAM1 proteins. Moreover, a dose-dependent effect of miR-126 alleviated the nerve tissue injury as well as reduced functional deficits [9].

Interestingly, application of crocetin, a natural carotenoid dicarboxylic acid, stimulated angiogenesis in traumatized rat brains. It occurred by means of an increased expression of VEGFR-2 and SRF genes with a concurrent inhibition of nerve cell apoptosis [1]. Of late it has been observed that GDF11 has a powerful proangiogenic influence on the artificially-induced stroke in rats. This neuroprotective effect is achieved through activin-like kinase 5 (ALK5), which increases proliferative potential of endothelial cells [16]. It turned out that osteopontin, an extracellular matrix protein, promoted angiogenesis and protected the nerve tissue from further damage. This effect is mainly produced by the protein's RDG and SLAY domains that contain certain sequences of amino acids [14]. It was also suggested that interleukin (IL)-1 $\alpha$ was a potent mediator of endothelial activation and a considerable marker of angiogen- esis, and played a significant role in reparative processes in the ischemic brain [22]. Ischemia-induced focal angiogenesis in the rat brain is more intensive in young animals than in old ones [25].

So far there have been few attempts to apply angiogenesis and its factors to medicolegal practice. They encompassed estimation of the age of the myocardial infarction, differential diagnosis of the septic shock and determination of the age of injuries to the integument $[8,10,11,17,18]$.

In our previous article, we verified usefulness of morphometric evaluation of neurofilaments, structural proteins of the nerve cell, for the determination of the age of brain contusions in human postmortem material [13]. The present paper continues these studies. It is aimed to examine a possibility of angiogenesis-related assessment of the age of brain contusions by means of morphometric evaluation of the CD34 antigen expression in blood vessels in contused sites among victims who died at different times after the brain trauma.

\section{Material and methods}

Neuropathological examinations were performed on paraffin blocks containing specimens of contused cortical-subcortical regions of cerebral hemispheres. All diagnoses of the brain contusion were made during medicolegal autopsies that were carried out in accordance with the Criminal Procedure Code. The researched material consisted of specimens from 90 deceased persons whereas the comparative material comprised specimens of 10 death cases due to sudden cardiac arrest. All paraffin blocks had been stored in the Department of Forensic Medicine of the Medical University of Gdańsk.

Police investigation teams worked out circumstances of each instance of the head trauma. In every case, the head trauma had been sustained as a result of either a car accident (a pedestrian, driver or passenger), a fall from height, an uncontrollable fall from erect position, or an assault.

The researched material was divided into nine groups according to the time of death: immediately on the spot, 12 hours, 24 hours, 2 days, 3 days, 4 days, 5 days, 6 days, and 7 days after the head trauma. Each group consisted of 10 cases. Persons who did not die instantly were all treated in hospitals.

After fixation in 4\% neutral buffered formaldehyde solution, the brain specimens were processed 
routinely and embedded in paraffin blocks. These were sliced into 4-micron-thick sections in the SM 2000R Leica microtome and stained with hematoxylin and eosin. Afterwards, the specimens were assessed in light microscopy so that representative material could be allotted to immunohistochemical examination. Paraffin slices were then mounted on glass slides and incubated in $36^{\circ} \mathrm{C}$ for 24 hours. The antigen retrieval was performed with a high-temperature epitope revealing heat-induced epitope retrieval (HIER) method in the Dako En Vision Flex Target Retrieval Solution ( $\mathrm{pH}$ 9). The process was carried out in the Dako PTLink machine. Immunohistochemical reaction was performed using the DAKOFLEX Monoclonal Mouse Anti-Human CD34 Class II Clone QB End 10 Ready-to-Use. Staining took place in the Dako Autostainer Link 48. Eventually, the specimens were dehydrated through a series of alcohol solutions of ascending concentrations, passed through xylene and enclosed in Canada balsam.

To evaluate time-dependent progression of lesions, the Met-llo application, developed at the Institute of Materials Science of the Silesian University of Technology [24], was used for morphometric analysis. Usability of the above-mentioned software was confirmed by our previous study [13]. Full-colour photomicrographs, taken at 200-fold magnifications with the AxioCam Erc $5 \mathrm{~s}$ camera, were evaluated in the Carl Zeiss Scope A1 light microscope. The area of the analyzed region was approximately 254762 square micrometers. In each case, four such regions were analyzed. To facilitate the analysis of biostruc- tural images functionalities of the Met-llo application were expanded with additional modules.

The analysis concerned the reaction between the antibody and the CD34 antigen. However, in every histological specimen of the nerve tissue, there could be seen other structures which were due to image artifacts produced in the course of tissue preparation and image digitalization. They represented what we may call information noise. Therefore, before the start of the morphometric analysis it was necessary to select only such pixels that represented the analyzed structures, i.e. antibody-CD34 antigen reactions. The end result of data processing was a binary image in which pixels of the analyzed structures were assigned a value of 1 and pixels of the background (not to be analyzed) were assigned a value of 0 . Eventually, the binary image was displayed as an overlay of the input image in such a way that zero-value pixels are transparent and pixels with value 1 have a blue colour (Fig. 1).

A researcher can perform binarization either manually or automatically on the basis of data contained in the grey level histogram of an analyzed image. As the cases in which binarization immediately yields a correct binary image of analyzed structures are rare, the detection process is usually divided into distinct phases:

- modification of the input image,

- binarization,

- modification of the binary image.

In individual specimens, displayed structures differ in grey-scale and colour. Moreover, some spec-
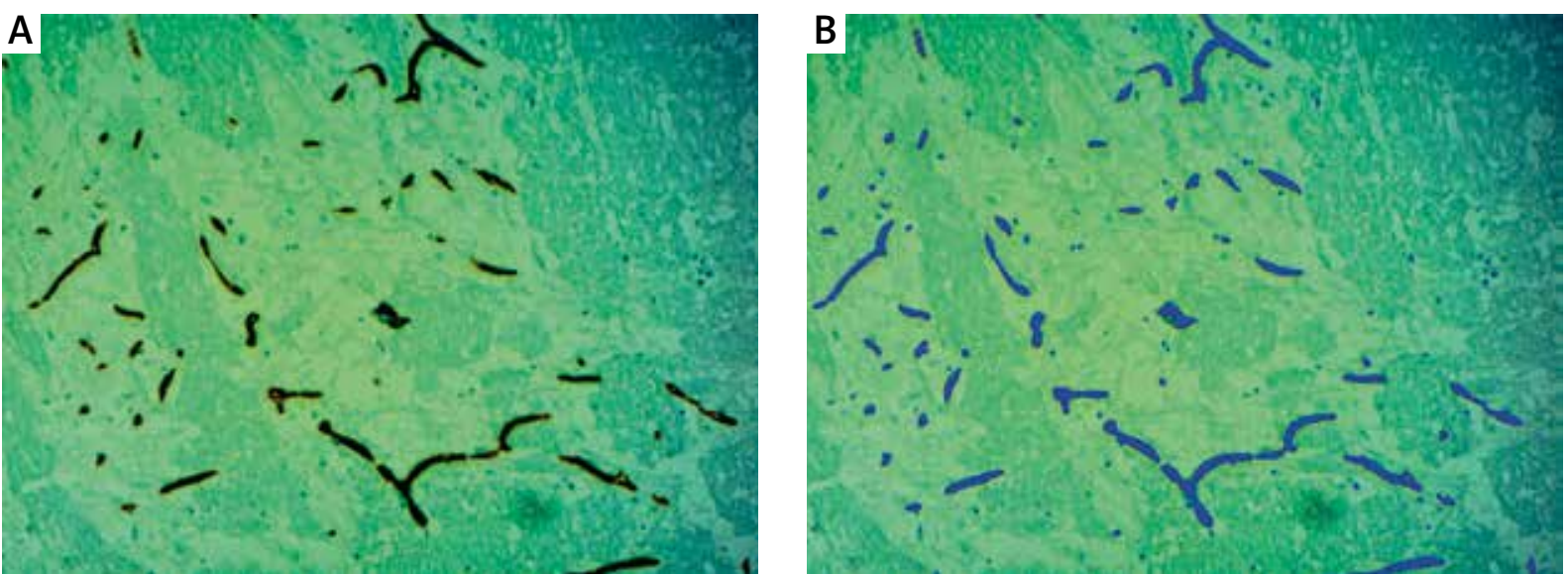

Fig. 1. Input microscopic image (A) and input microscopic image overlaid with the binary image of analyzed structures (B). 

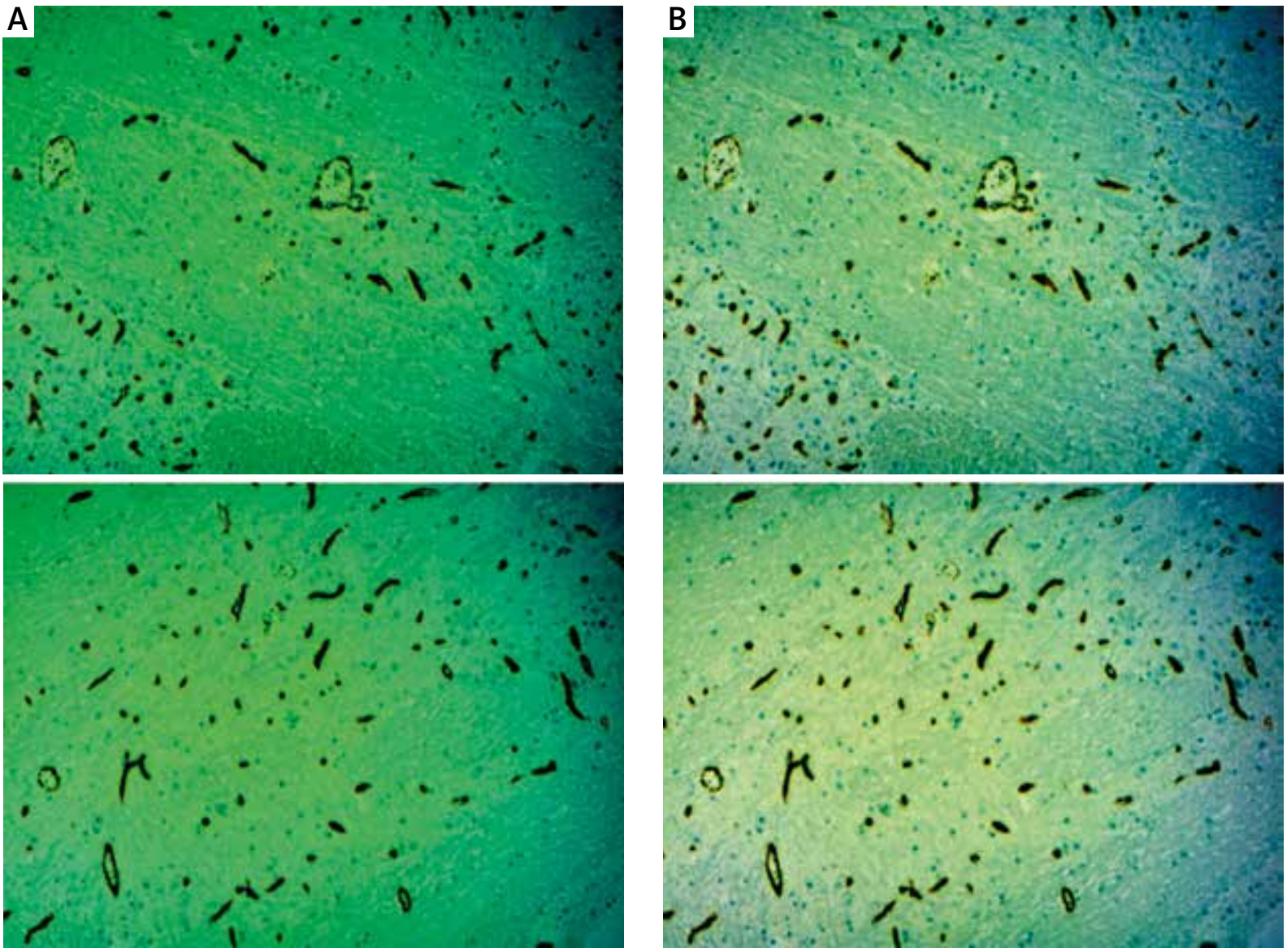

Fig. 2. Input images (A) and input images after R, G and B channel histogram normalization (B).

imens can be unevenly illuminated (the so-called shade effect). This artifact is represented by darker areas in peripheral sections of an image (Fig. 2). In order to develop a universal method of detection and quantitative description of blood vessels one must minimize the above-mentioned discrepancies. The first defect, i.e. differences in grey-scale and colour, can be removed by normalization of greyscale histograms of the channels $R, G$ and $B$ in input images. Albeit not easily noticeable, the effect is very important for next stages of blood vessels detection (Fig. 2). However, normalization does not eliminate the second flaw, i.e. shading which negatively affects blood vessels detection (Fig. 3A). Therefore, normalized input images are specifically processed so that shading is removed before binarization. The result of this procedure can be seen in Figure 3B.

The analyzed structures variably bind labelled antigen CD34 antibodies. To objectify evaluation, we decided that only intensely-stained structures would be assessed quantitatively. Preliminary examinations suggested that the highest-quality binary images could be obtained with the Ridler and Calvard k-means method (Fig. 3B) [20]. In this method, a histogram is divided into two areas representing pixels of the grey level lower and higher than the threshold value $\mathrm{k}$.

Output value of this threshold equals the arithmetic mean of minimal and maximal grey levels of the analyzed image. Each of these two areas is assigned a mean grey level (g1 and g2, respectively) and a new threshold value $\mathrm{k}$ is calculated using the following formula: $k=(g 1+g 2) / 2$. This value is used to divide the histogram into two new separate areas. The procedure is repeated with thresholds $\mathrm{k}$ computed in subsequent measuring loops till the threshold value $\mathrm{k}$ becomes constant.

The whole procedure was fully automated and manual correction of binary images was rarely needed. Detection of blood vessels and measurement of their geometrical characteristics were performed 

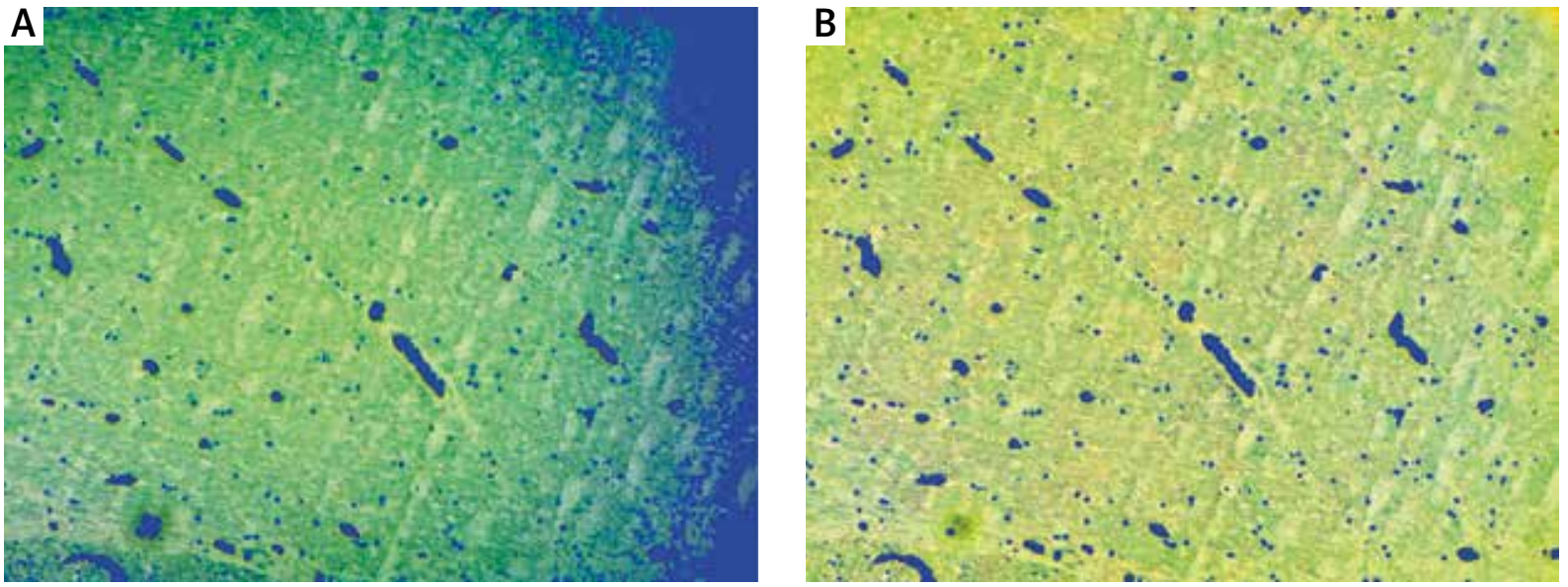

Fig. 3. Binary images of blood vessels (blue areas) after histogram normalization performed on input microscopic images (A) and after histogram normalization and removal of shading (B).

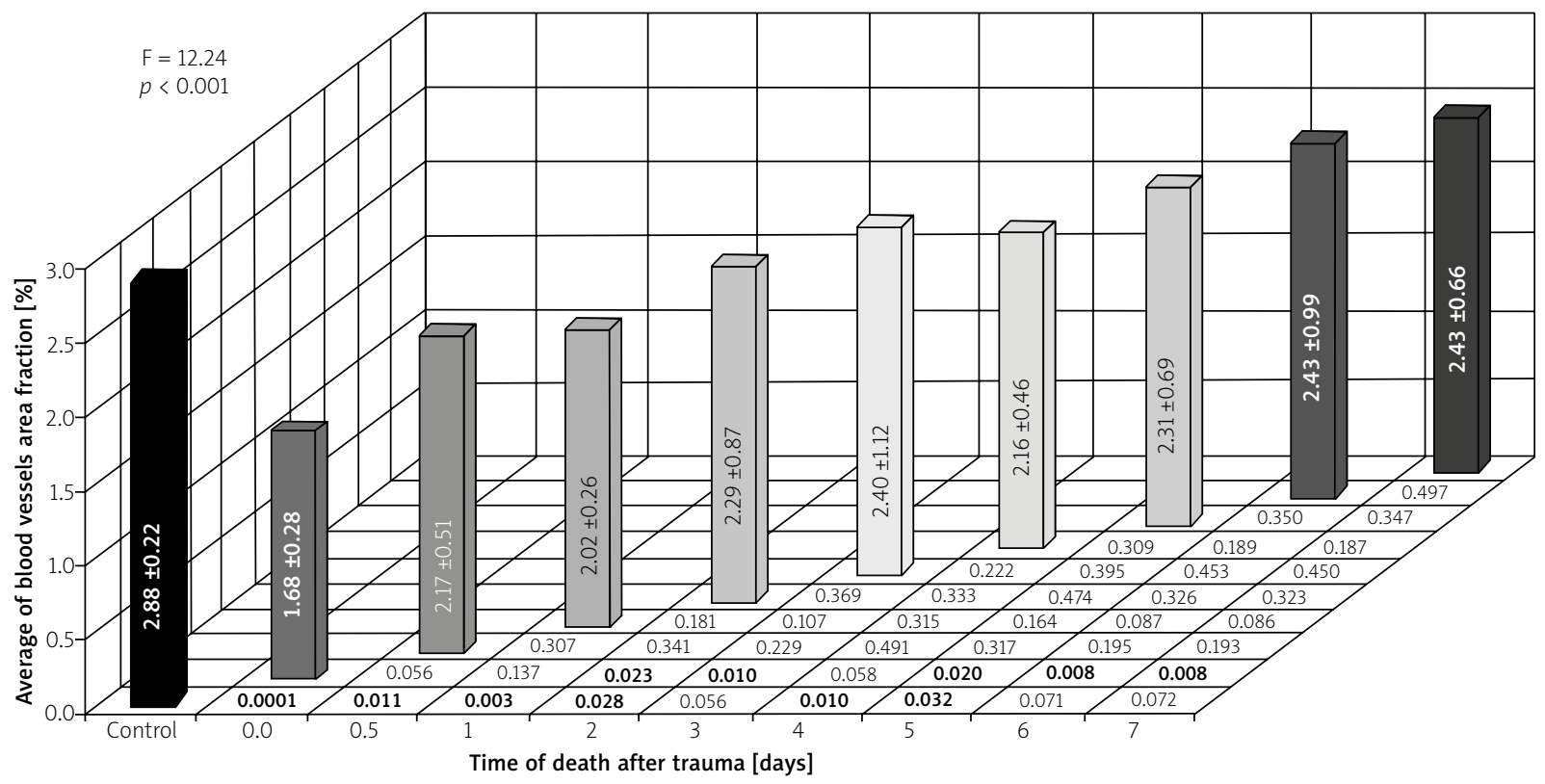

Fig. 4. The relationship between the area fraction of blood vessels and the time after head trauma. The results of the Fisher NIR test are at the bottom of the bar chart. Statistically significant differences are bolded.

by the Met-Ilo application. The surface method was applied to measurement, all available magnitudes being calculated. Additionally, structures representing blood vessels which counted less than ten pixels were left out as they might be artifacts. These structures were deleted from binary images.

The results underwent statistical analysis. To characterize quantitatively blood vessels, two features were chosen: the area fraction and the number in an analyzed field. Hypotheses that both features are normally distributed were verified with the Shapiro-Wilk test. The result was positive for the area fraction and oneway analysis of variance (ANOVA) could be conducted. However, as the second feature was not normally distributed, the non-parametric Kruskal-Wallis test had to be applied. The results of analysis of variance are shown in Figures 4 and 5. Mean standard deviations values (for area fractions) and the first, second and third quartiles (for numbers of blood vessels) are given. In both cases, there were significant differences of 


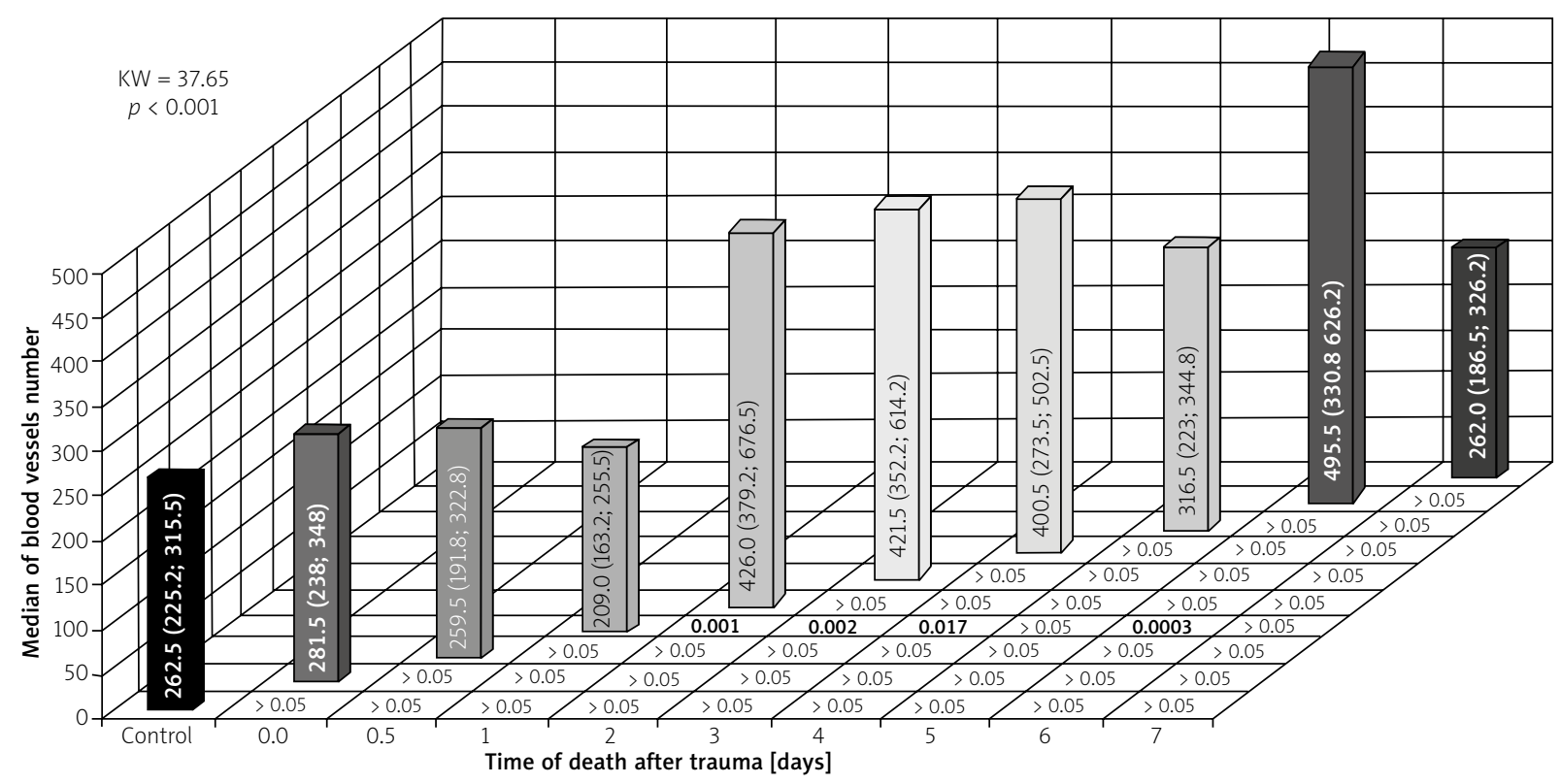

Fig. 5. The relationship between the number of blood vessels and the time after head trauma. The results of Dunn's test are at the bottom of the bar chart. Statistically significant differences are bolded.

values $(p<0.05)$. Post-hoc analyses were conducted: the least significant difference test (Fisher NIR) for area fractions and Dunn's test for numbers of blood vessels.

\section{Results}

Generally, there was a tendency to increase both the number of blood vessels and blood vessels area fraction in time.

Figure 6 shows examples of final binary images. Figures 4 and 5 display the results of statistical analysis of morphometric studies on CD34 protein expression. Figure 4 shows the relationship between the area fraction of CD34-expressed blood vessels (i.e. their density) and the time after head trauma. Figure 5 presents the relationship between the number of CD34-expressed blood vessels and the time after head trauma.

The non-parametric Kruskal-Wallis test (Fig. 5) implies that numbers of blood vessels are significantly different $(\mathrm{KW}=37.65, p<0.001)$ at each examined time after death. Significance levels, calculated with Dunn's test, suggest that:

- the result of Kruskal-Wallis' test is a consequence of significant differences between observations on day 1 and observations on days 2, 3, 5 and 6,

- specific sample pairs are not statistically significant,
- a small number of cases featuring significant differences is a consequence of dispersion of numbers of blood vessels, this conclusion being supported by values of interquartile ranges.

\section{Discussion}

The VEGF protein family $(-A,-B,-C,-D,-E)$ plays a key role in both angiogenesis and vasculogenesis. It mainly influences endothelial cells lining internal surfaces of the cardiovascular system. It stimulates their proliferation and migration, and promotes synthesis of extracellular matrix proteases which enables neoangiogenesis $[3,27]$. At present, thanks to immunohistochemistry, endothelial cells can be labelled and identified in body tissues with surface markers, e.g. CD34, CD31, vWF antigens. To this end, the CD34 antigen is most commonly employed (Gp105-120) [11].

The CD34 antigen is a transmembrane protein with the molecular mass of $115 \mathrm{kDa}$, which participates in adhesion, i.e. mutual attachment of endothelial cells, and their migration during neovascularization [23]. This antigen is present in the membrane of several hematopoietic stem cells in bone marrow. The CD34 expression is observed in initial stages of development of endothelial progenitor cells (EPCS) and in early phases of formation of new blood ves- 

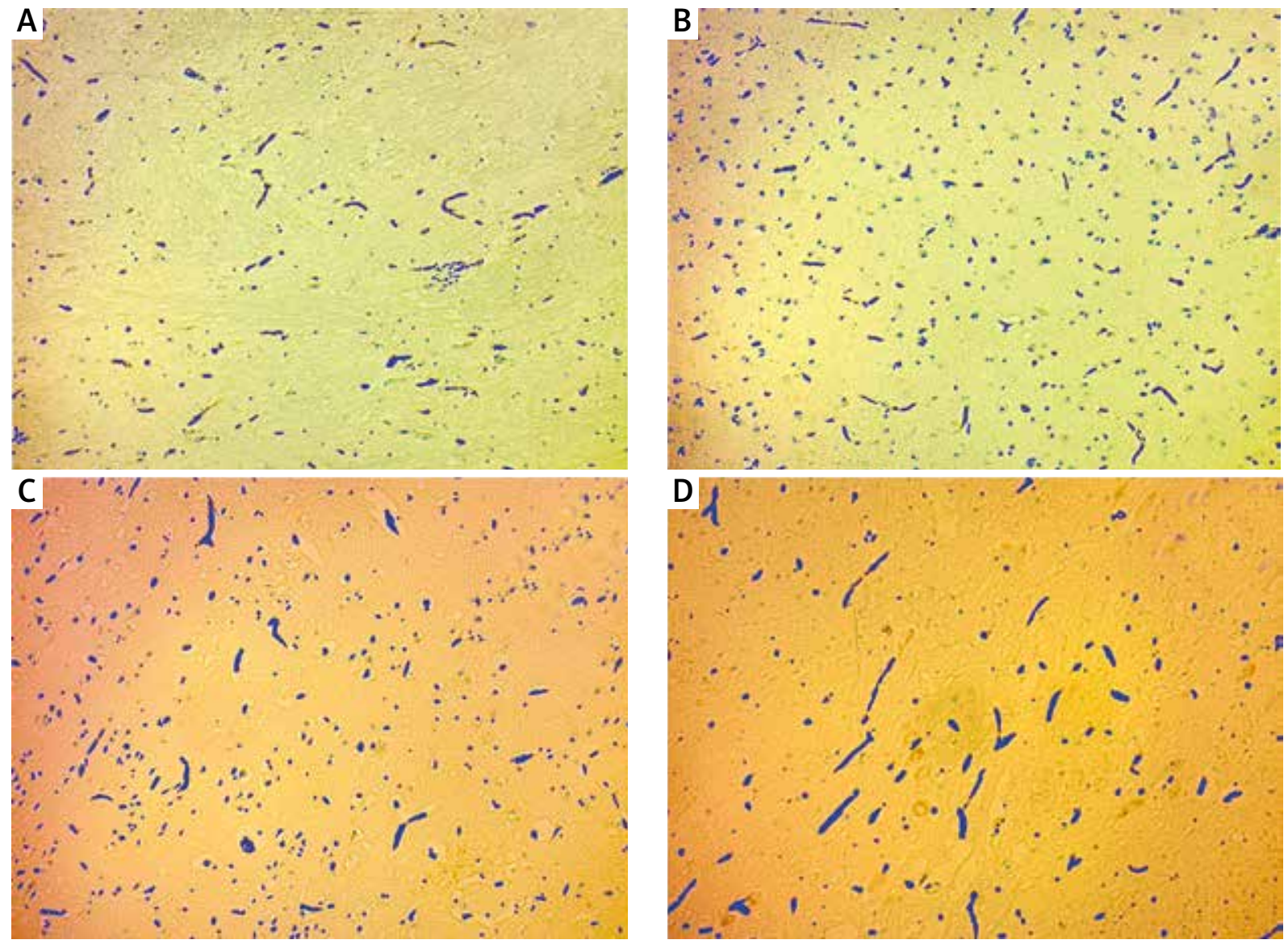

Fig. 6. Examples of final binary images of blood vessels (blue areas) showing focal brain contusions. Victims died immediately on the spot (A), 12 hours (B), 2 days (C) and 7 days (D) after head trauma.

sels [11]. As EPCs develop and mature, the CD34 expression on their surface decreases. Therefore, it is commonly used as a marker of neovascularization to assess their density in tumors. Having taken into account its characteristics, we have decided to employ the CD34 antigen in our study.

As mentioned in the introduction, there have been few attempts to apply angiogenesis and its factors to medicolegal practice. Moreover, we have not come across many studies concerning angiogenesis in the brain contusion in humans. In Gömöritrichrome-stained specimens, Oemichen found out proliferating blood capillaries in the proximity of contusions in brains of the victims who had died in the second half of the first week after brain trauma [19]. This finding is confirmed by two fundamental handbooks on forensic neuropathology. According to their authors, 5-7 days after the brain trauma sprouting blood capillaries begin to penetrate the site of a brain contusion while the injured tissue is still being phagocytosed. This process reaches its plateau in the third week and then decreases over months and years $[12,15]$.

So far Hausmann and Betz have conducted the most extensive research on application of immunohistochemistry in determination of the age of brain contusions [7]. They studied expression of extracellular matrix proteins and the basement membrane, which are involved in vascular repair and formation of new blood vessels in injured areas. The proteins they examined were: laminin, type IV collagen, tenascin, and markers of endothelial cells - thrombomodulin and factor VIII. The researched material comprised brains of the deceased due to fatal brain contusions, the control group consisted of brains of people whose death was not related to trauma. Laminin and type IV collagen stainings were positive in both groups and, thus, they were considered irrel- 
evant. The immunoreactivity of tenascin, thrombomodulin and factor VIII was higher in the proximity of cortical contusions when compared with the control group. A significantly increased expression of factor VIII was detected 3 hours after head trauma, of tenascin - after 1.6 days, of thrombomodulin - after 6.8 days, and persisted, respectively, for 6 days- 4 weeks, 1-4 weeks and 1-2 weeks after the infliction of injuries [5-7]. According to the authors, further studies on tenascin and thrombomodulin are desired. On the contrary, usability of factor VIII was considered doubtful, especially shortly after trauma, because it also reacted to some extent with endothelial cells in the control group.

The above results of studies on and statements about relationships between different stages of angiogenesis in immediate surroundings of brain contusions and the time since trauma are not sufficient to use these methods in everyday medicolegal practice. They require further confirmation. The authors emphasize it, as well. Our morphometric method of automatized assessment of the CD34 expression in brain contusions represents an innovative approach. It can be employed for verification of other candidates for reliable biomarkers of the age of such injuries.

To sum up, while the results of Kruskal-Wallis analysis allow to conclude that numbers of blood vessels are significantly different in the first seven days after the head trauma, significance levels of specific sample pairs, as calculated by Dunn's test, are not statistically significant. Thus, morphometric analysis of the CD34 expression in the contused sites has shown that the proposed method is of no considerable value in determination of the age of brain contusions as opposed to morphometric analysis of neurofilaments [13]. This is caused by major disparities between dynamism of angiogenesis and dynamism of synthesis of structural proteins of nerve cells. The formation of new blood vessels, both in physiological and pathological conditions, requires activity of a higher number of tissue-regulating factors than processes of neurofilament degradation and polymerization. It seems that sensitivity of nerve cells to all kinds of injuring factors is greater than that of other types of body cells. Therefore, further studies on other biomarkers of the age of brain contusions should be conducted. Perhaps it will be eventually possible to design a panel of antigens which can be used in everyday medicolegal practice.
What is important, while interpreting results of this kind of studies, one must remember about non-traumatic causes of neovascularization in the brain, such as long-term effects of ischemia in arterial hypertension and atherosclerosis of cerebral arteries as well as at the site of an earlier stroke, which requires differentiation between "post-infarct" and "post-traumatic" angiogenesis $[21,26]$. Their presence can pose a serious problem in medicolegal expert practice. However, we would like to emphasize the uniqueness of this coincidence. In our studies, which included a few hundred randomly chosen cases, we have not stumbled across a single instance of this kind.

\section{Disclosure}

The authors report no conflict of interest.

\section{References}

1. Bie $X$, Chen $Y$, Zheng $X$, Dai $H$. The role of crocetin in protection following cerebral contusion and in the enhancement of angiogenesis in rats. Fitoterapia 2011; 82: 997-1002.

2. Dettmeyer RB. Forensic histopathology. Springer-Verlag, Berlin Heidelberg 2011.

3. Dulak J, Józkowicz A, Łoboda A. Angiogenesis and vascularisation. Cellular and molecular mechanisms in health and diseases. Springer-Verlag, Wien 2013.

4. Folkman J. Tumor angiogenesis: therapeutic implications. N Engl J Med 1971; 285: 1182-1186.

5. Hausmann R. Timing of cortical contusions in human brain injury. morphological parameters for a forensic wound-age estimation. In: Forensic pathology reviews. Vol. 1. Tsokos M (ed.). Humana Press Inc, Totowa, New Jersey 2004; 53-75.

6. Hausmann R. Age determination of brain contusions. Forensic Sci Med Pathol 2006; 2: 85-93.

7. Hausmann R, Betz P. The time course of the vascular response to human brain injury - an immunohistochemical study. Int J Legal Med 2000; 113: 288-292.

8. Hayashi T, Ishida Y, Kimura A, Takayasu T, Eisenmenger W, Kondo T. Forensic application of VEGF expression to skin wound age determination. Int J Legal Med 2004; 118: 320-325.

9. Hu J, Zeng L, Huang J, Wang G, Lu H. miR-126 promotes angiogenesis and attenuates inflammation after contusion spinal cord injury in rats. Brain Res 2015; 1608: 191-202.

10. Ishida Y, Kimura A, Takayasu T, Eisenmenger W, Kondo T. Expression of oxygen-regulated protein 150 (ORP150) in skin wound healing and its application for wound age determination. Int J Legal Med 2008; 122: 409-414.

11. Ishikawa Y, Akishima-Fukasawa Y, Ito K, Akasaka Y, Tanaka M, Shimokawa R, Kimura-Matsumoto M, Morita H, Sato S, Kamata I, Ishii T. Lymphangiogenesis in myocardial remodelling after infarction. Histopathology 2007; 51: 345-353.

12. Itabashi HJ, Andrews JM, Tomiyasu U, Erlich SS, Sathyavagiswaran L. Forensic neuropathology. A practical review of the fundamentals. Academic Press, London, UK 2007. 
13. Kobek M, Jankowski Z, Szala J, Gąszczyk-Ożarowski Z, Pałasz A, Skowronek R. Time-related morphometric studies of neurofilaments in brain contusions. Folia Neuropathol 2016; 54: 50-58.

14. Lee H, Jin YC, Kim SW, Kim ID, Lee HK, Lee JK. Proangiogenic functions of an RGD-SLAY-containing osteopontin icosamer peptide in HUVECs and in the postischemic brain. Exp Mol Med 2018; 50: e430.

15. Leestma J. Forensic neuropathology. CRC Press Taylor and Francis Group, New York 2009.

16. Ma J, Zhang L, Niu T, Ai C, Jia G, Jin X, Wen L, Zhang K, Zhang O, Li C. Growth differentiation factor 11 improves neurobehavioral recovery and stimulates angiogenesis in rats subjected to cerebral ischemia/reperfusion. Brain Res Bull 2018; 139: 38-47.

17. Manoilescu I, Teleman S, Cojocaru E, Mihăilă D, Plămădeală $P$. Vascular endothelial growth factor (VEGF) expression in the lung in toxic septic shock. Rom J Morphol Embryol 2011; 52 (1 Suppl): 309-313.

18. Nogami M, Hoshi T, Arai T, Toukairin Y, Takama M, Takahashi I. Morphology of lymphatic regeneration in rat incision wound healing in comparison with vascular regeneration. Leg Med 2009; 11: 213-218.

19. Oemichen M, Auer RN, Konig HG. Forensic neuropathology and associated neurology. Springer-Verlag, Berlin Heidelberg 2006

20. Ridler TW, Calvard S. Picture thresholding using an iterative selection method. SMC 1978; 8: 629-632.

21. Salehi A, Zhang JH, Obenaus A. Response of the cerebral vasculature following traumatic brain injury. I Cereb Blood Flow Metab 2017; 37: 2320-2339.

22. Salmeron K, Aihara T, Redondo-Castro E, Pinteaux E, Bix G. IL-1alpha induces angiogenesis in brain endothelial cells in vitro: implications for brain angiogenesis after acute injury. J Neurochem 2016; 136: 573-580.

23. Sidney LE, Branch MJ, Dunphy SE, Dua HS, Hopkinson A. Concise review: evidence for CD34 as a common marker for diverse progenitors. Stem Cells 2014; 32: 1380-1389.

24. Szala J. Application of computer-aided image analysis methods for quantitative evaluation of material structure (in Polish) Silesian University of Technology, Gliwice 2001.

25. Tang Y, Wang L, Wang J, Lin X, Wang Y, Jin K, Yang GY. Ischemia-induced angiogenesis is attenuated in aged rats. Aging Dis 2015; 7: 326-335.

26. Yin KJ, Hamblin M, Chen YE. Angiogenesis-regulating microRNAs and Ischemic Stroke. Curr Vasc Pharmacol 2015; 13: 352 365.

27. Zadeh G, Guha A. Angiogenesis in nervous system disorders. Neurosurgery 2003; 53: 1362-1374; discussion 1374-1376. 